DESIGN CHARTS FOR VACUUM PLATES

\author{
J. E. O'Meara
}

January 1986 


\title{
DESIGN CHARTS FOR VACUUM PLATES
}

\author{
J. E. O'Meara
}

January 1972

\section{INTRODUCTION}

This paper presents design charts to aid designers in selection of a wall thickness for flat plates used on vacuum vessels.

These charts display both stress and deflections for a given wall thickness and plate dimensions. Since both stress and deflection are plotted on a single graph, one may select the proper wall thickness quite readily.

Precautions for use of the charts are also presented.

\section{DESCRIPIION OF DESIGN CHARTS}

A recurring design problem encountered by vacuum vessel designers is the selection of a plate thickness for a vacuum vessel wall.

Often a plate thickness is selected based upon what "looks about right". In many applications, the designer must consider deflections as well as the stress. The writer has prepared design charts for circular plates and rectangular plates under a pressure differential of one atmosphere. 
The edge constraint for plates may be either fixed or simply supported. Most practical designs have edge conditions that are neither fixed nor simply supported, therefore, the designer should check both the fixed edge chart and the simply supported edge chart to make certain that a safe design has been obtained.

The equations used have all been selected from R. J. Roark. (I) Each chart shows a shaded area. In this shaded area the plots are not completely valid. The plates behave as diaphrams in this area and the deflections and stresses are somewhat less than shown. In other words, if one uses the chart as is, it would result in a safe design in that the stresses would be below those shown.

If one must select extremely thin plates for a chamber, a more intricate design study should be made based upon diaphram stresses rather than use these charts.

All charts assume a Young's modulus of $30 \times 10^{6}$ psi. While the stresses shown on the plots are valid for every Young's moduli, the deflections are not. When using a material with a different Young's modulus, one must multiply the chart deflections by the following ratio:

$$
\frac{E_{30 \times 10^{6}}}{E_{\text {material }}}
$$

(1) Roark, R.J. Formulas for Stress And Strain 
The rectangular plate charts show "safe" values. In other words, the plotted values are for $b$ to a ratio of zero. Most designs have $\mathrm{b}$ to a ratios considerably more favorable than this. The plotted values can be corrected by using the chart in the upper left hand corner. Stresses and deflections may be reduced, in the case of square plates by as much as a factor of 3. However, it is pointed out that failure to use these "correction factors" results in a conservative design.

\section{PRECAUTIONS}

In summary, the following precautions should be noted:

1. One should check both the fixed edge and simply supported edge charts prior to selecting a wall thickness.

2. For young modulus, other than $30 \times 10^{6} \mathrm{psi}$, apply the following correction factor for the deflections shown:

$$
\frac{E_{30 \times 10^{6}}}{E_{\text {material }}}
$$

3. No Young's modulus correction is reçuired for stresses.

4. In the use of rectangular plates, one may reduce the stresses and deflections by using the correction chart in upper left hand corner.

5. In unusual applications, where extremely thin windows are to be used, one should consult diaphram equations. 
6. The plotted values are valid for deflections up to $y=1 / 2 t$. They are conservative for greater deflections.

7. The chart shows deflections at the plate center. In some applications, deflections at other locations cause the loss of 0 -ring sealing pressures or similar problems. In such applications, the designer should do further analysis.

The following examples demonstrate the use of these charts:

\section{Example 1:}

Select plate thickness for circular plate 9" diameter, fixed edges $E=30 \times 10^{6}$. Maximum allowable stress is 20,000 psi.

Enter chart $d=9 "$

Select $t=1 / 8^{\prime \prime}$

Read stress 15,000 psi

Read deflection .019"

\section{Example 2:}

Select plate thickness for $7 "$ x $10 "$ plate. Rectangular plate. Fixed edges. $E=30 \times 10^{6}$. Maximum allowable stress is 20,000 psi.

Enter correction chart at $\quad \frac{b}{a}=\frac{7^{\prime \prime}}{10^{\prime \prime}}=.7$

Read $C_{S}=.85$

$$
c_{d}=.8
$$

Enter large chart at $\mathrm{b}=7 "$

Select $t=1 / 8^{\prime \prime}$

Read stress $22,500 \times \mathrm{C}_{\mathrm{S}}=19,125 \mathrm{psi}$

Read deflection .016 $\times \mathrm{C}_{\mathrm{d}}=.013 "$ 
I wish to acknowledge the efforts made by Alexander Baron in checking the accuracy of these charts. 


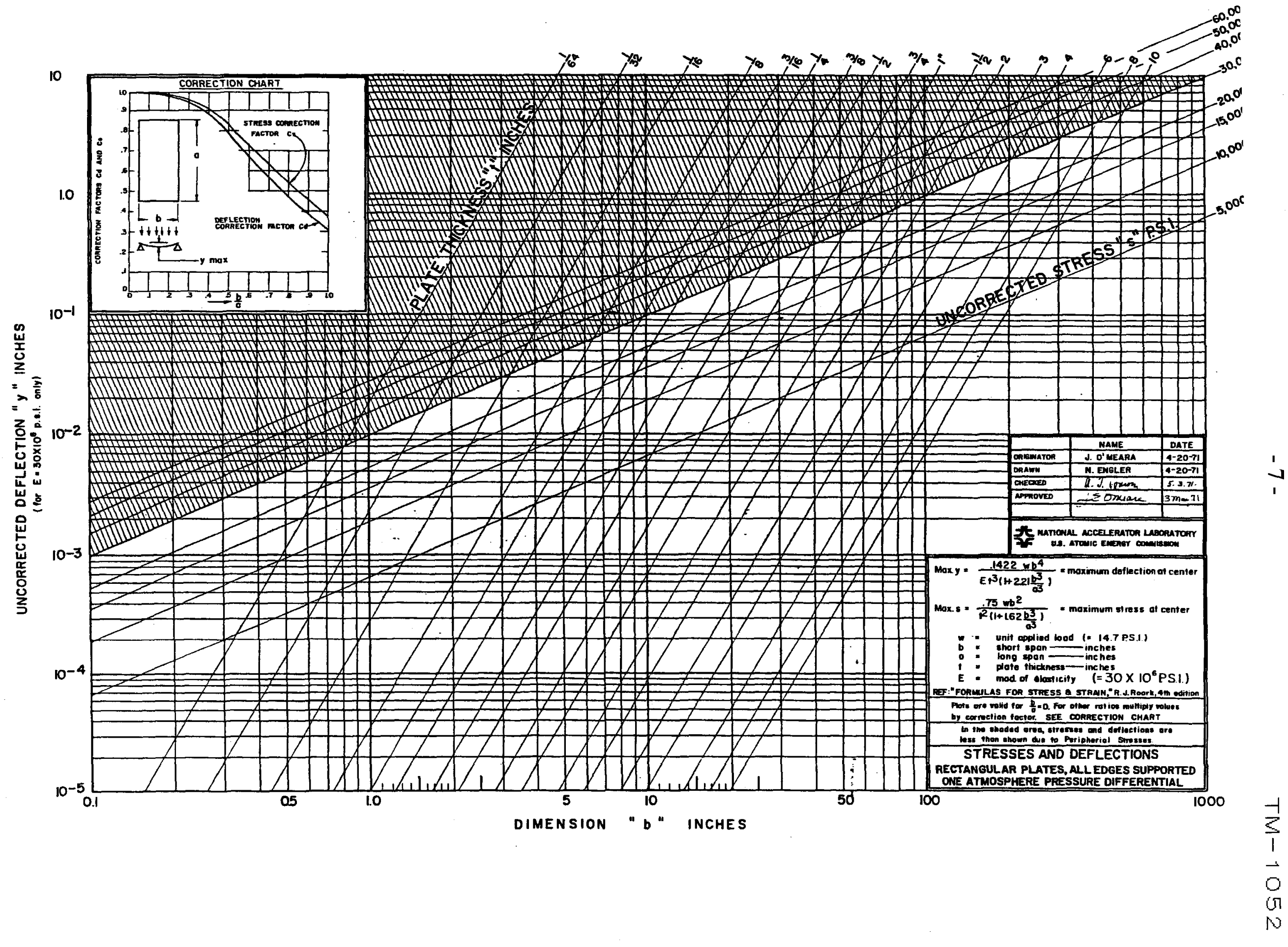

